\title{
Effect of microwave pretreatment on dyeing performance of wool fabric
}

\begin{abstract}
An investigation was undertaken to study microwave pretreatment and dying process of wool fabric. Microwave pretreatment of wool fabric was carried out under variety of conditions in terms of the use of water, $50 \mathrm{~g} / \mathrm{L}$ urea, $100 \mathrm{~g} / \mathrm{L}$ urea in the treatment solution. The area shrinkage, surface morphology, crystallinity and dye ability of the pretreated wool fabric were measured. Effects of microwave fixation time and batching time before microwave fixation on $\mathrm{K} / \mathrm{S}$ value were analyzed. Fixation, uniformity, color fastness, breaking strength and breaking extension of wool fabric were compared among cold batching fixation, microwave fixation and microwave fixation after pretreatment.
\end{abstract}

Keywords: wool fabric, microwave, pretreatment, dyeing, reactive dyes, urea
Volume I Issue 6 - 2017

\author{
Zhao Xue , $^{1,2}$ \\ 'Shaoxing University, China \\ ${ }^{2}$ Clean Dyeing and Finishing Technology Research Laboratory of \\ Zhejiang Province, China
}

Correspondence: Zhao Xue, College of Textile and Garment, Shaoxing University and Clean Dyeing and Finishing Technology Research Laboratory of Zhejiang Province, Shaoxing 3|2000, China,Tel I3605859763, Email zhaoxue44455709@sina.com

Received: April 02, 2017| Published: May 10, 2017

\section{Introduction}

The problems in producing a reactive dye for wool lie in wool's complex structure. The former dyeing process leads to the requirement for long continuous dyeing times at elevated temperature; under these conditions the dye tends to react before levelness is achieved. Numerous studies have been made of methods of simplification of the dyeing process to reduce the time and temperature of processing. The use of high efficient dyeing methods to achieve energy savings and minimize wool damage has been the subject of considerable.

Microwaves are high frequency radio waves which are capable of penetrating many materials and causing heat to be generated in the process. The electric field energy is converted into heat through the dielectric losses of the material. Materials which absorb microwaves are thus described as "lossy". The greatest dielectric losses occur when the molecules of the substance irradiated are dipolar (e.g., $\mathrm{H}_{2} \mathrm{O}$ ). In the presence of the high frequency electromagnetic field the molecules oscillate synchronously with it, and this causes heat to be generated through inter-molecular friction..$^{1-3}$ The textile Industry has investigated many uses for microwave energy from heating, drying, dye fixation, printing and curing of resin-finished fabrics. In 1966, Ciba-Geigy obtained one of the earliest patents for using microwave heating in dyeing and printing fibrous material with reactive dyes. Since then, many authors have investigated the feasibility of using microwaves for variety of dyeing and finishing processes. However, the potential of using microwave radiation as a heating source for dye fixation directly after pad application of dye bath to the fabric has not been investigated widely. Research in this area has been confined to limited textile substrates, dyes and solvent systems. Few studies have investigated the feasibility of using microwaves to dye pretreated wool fabrics with reactive dyes. ${ }^{4-6}$

The present work was undertaken with a view toward studying the characteristics of the dyeing process of microwave-pretreated and untreated wool fabric by microwave fixation and untreated wool fabric by cold batching fixation with reactive dyes. The study also included the physical changes in wool structure brought about by microwave pretreatment using X-ray diffraction analysis and optical microscope examination.

\section{Experimental}

\section{Materials}

The fabric used was a weave $100 \%$ wool fabric. Lanasol reactive dyes; Urea; sodium bi sulfite; acetic acid; ammonia; Peregal O.

\section{Procedure of microwave pretreatment of wool fabric}

a. Soaking the wool fabrics in water, $50 \mathrm{~g} / \mathrm{L}$ urea and $100 \mathrm{G} / \mathrm{L}$ solution at ambient temperature, until the fabrics were completely wetted;

b. Heating the fabrics in the microwave oven at $700 \mathrm{~W}$ for different intervals of time (1-5 minutes).

c. After pretreatment, taking the water and urea treated fabrics out; the samples were picked up from the water and urea, sucked into a Buckhner flask, centrifuged and then dried at room temperature for a lengthy period.

\section{Procedure of pad-dyeing}

The basic dyeing pad liquor consisted of:

i. Lanasol reactive dye $2.5 \mathrm{~g} / \mathrm{L}$

ii. Peregal O $15 \mathrm{~g} / \mathrm{L}$

iii. Urea $100 \mathrm{~g} / \mathrm{L}$

iv. sodium bi sulfite $20 \mathrm{~g} / \mathrm{L}$

The wool textile to be dyed is padded at about room temperature with the padding liquor which contains dye, peregal $\mathrm{O}$, urea, and sodium bi sulfite. The liquor pickup can be $100 \%$ on weight of dry fiber.

Procedure 1: Thereafter, heating the fabrics in the microwave oven at $700 \mathrm{~W}$ for different intervals of time (1 10minuites) to fix the dyestuff.

Procedure 2: The moist cloth is batched at about room temperature for 24hours, which is customarily effected in the rolled-up state by slowly rotating the beam to fix the dyestuff. 
Procedure 3: The moist cloth is batched at about room temperature for different intervals of time (10 60minuties), which is customarily effected in the rolled-up state by slowly rotating the beam, then heating the fabrics in the microwave oven at $700 \mathrm{~W}$ for 6 minutes to fix the dyestuff.

Advantageously this measure subsequently the completed dyeing is first rinsed with water at $40^{\circ} \mathrm{C}$, to remove dye portions not fixed to the substrate and nonreactive dye portions formed by hydrolysts from the lanasol reactive dye is washed at $80^{\circ} \mathrm{C}$ by treatment with ammonia in an alkaline medium at $\mathrm{pH} 8.5$ for 15 minutes, is rinsed once more with water and is then neutralized with acetic acid.

\section{Fabric performance evaluation}

The breaking strength and elongation of the fabric is measured according to ASTMD 5034. Colorfastness to Laundering of the fabric is measured according to AATCC Test Method 61-2007. Colorfastness to Crocking of the fabric is measured according to AATCC $8 . \mathrm{K} / \mathrm{S}$ values as calculated from the Data color SF600 color measuring and matching instrument are used to determine the shade depth of dyed wool fabrics. Uniformity of dyeing is evaluated from the Data color SF600 color measuring and matching instrument by measuring average deviation $\mathrm{S}$, range $\mathrm{P}$ of the maximum and the minimum for $\mathrm{L}$, $\mathrm{C}$ and $\mathrm{H}$ and balanced color difference $\Delta \mathrm{E}$ at specified twenty uniform locations on the wool fabrics. Fixation is determined from the Data color SF600 color measuring and matching instrument by measuring ratio of K/S for wool fabrics which are rinsed, washed, neutralized and then dried and wool fabrics which are dried after fixation without washing. Shrinkage test: shrinkage due to microwave pretreatment was estimated by measuring the changes in the dimensions of the piece sample: area shrinkage $(\%)=($ area before treatment-area after treatment $)$ /area before treatment $\times 100$. Various analyses of the surface morphology of the untreated and treated wool fabric were carried out with scanning electron microscopy (SEM). Crystallinity measurement of untreated and treated wool fabric was carried out with X-ray diffraction (XRD).

\section{Results and discussion}

The effects of microwave heating time on the dye ability of wool fabric

Procedure of pad-dyeing referred to 2.3 (Procedure 1 and procedure 2). Time of microwave heating was changed to study the effects of different microwave heating time on the dye ability of wool fabric. The result was shown in Table 1.

The dependence of the dyeing color depth on the microwave heating time is shown in Table 1. It can be seen, from Table 1, that the microwave heating time has a greater impact on the color depth of the dyed wool fabric, the longer the heating time, the greater the color depth of the dyed wool fabric, however, microwave heating time was increased to 6minutes, an increase in the heating time decreased color depth of dyed wool fabric which was caused by hydrolysis of reactive dye resulting from higher temperature using longer microwave heating time. The optimum conditions of treatment, based on the analysis were: heating in microwave oven at $700 \mathrm{~W}$ for 6 minutes. It can also been seen, from Table1, that microwave heating for 6 minutes produced better dye fixation results than those obtained after 24 hours batching at room temperature.

The rate of dyeing with microwave heating is much faster than cold pad batching. In the case of microwave heating equilibrium can be reached with a few minutes, where in the case of cold pad batching equilibrium is established after a few hours. Additionally, the effect of microwave radiation on the wool fabric itself suggests that microwave-induced oscillation of the dye molecules did occur, and that such oscillation aided the diffusion of the dyes into the wool fiber.

The oscillation of water molecules in the dye bath could promote dye diffusion. At room temperature, the molecules in liquid water are believed to be highly ordered in layers or clusters. Even at boil clusters of molecule still exist. This aggregation limits solute migration through water. Microwave radiation-induced oscillation of the water molecules in boiling water result in the break-up of these cluster to some extent, thus facilitating migration of solutes, and, in general increasing the movement of any solute through water and, ultimately, increasing the rate of diffusion. ${ }^{7}$

The principal advantages of the pad-batch-microwave system are thus:

Simplicity: No heating of the fabric before batching and no humidity control in the batching chamber (oven) are required. No preheating of the oven is required.

Flexibility: Batching does not have to take place inside the oven and batches can be left at room temperature, if necessary, unit an oven becomes available.

Efficiency: Microwave provides a means of a very efficient heating. Efficiencies of more than $70 \%$ are claimed for the conversion of mains electricity into heat energy when textile materials are heated by $900 \mathrm{MHz}$ microwaves. ${ }^{8}$

\section{The effects of batching time on the dye ability of wool fabric}

Procedure of pad-dyeing referred to 2.3 (Procedure 3). Batching time was changed to study the effects of different batching time before microwave heating on the dye ability of wool fabric. The result was shown in Table 2.

Table 2 represents the effect of batching before microwave fixation on the color depth of dyed wool fabric. It is obvious that the color depth of dyed wool fabric was increased when batching for period of time before microwave fixation, and the relative color strength $(\mathrm{K} / \mathrm{S})$ increases by increasing the batching time before microwave fixation.

To obtain deep level dyeing on wool fabric, the fiber could be swollen by cold batching for period of time before microwave fixation. Upon exposure to microwave radiation, the additional localized energy from oscillation of the water molecules inside wool fabric may increase the breaking of interaction bonds, causing the internal structure to be further opened to greater dye diffusion.

\section{The effects of microwave pretreatment}

The effects of microwave pretreatment on the area shrinkage of wool fabric: Procedure of pretreatment referred to 2.2. Pretreatment process was changed to study the effects of pretreatment before microwave heating on the area shrinkage of wool fabric. The result was shown in Table 3.

Table 3 showed that the tendency of wool fabric used was to shrink when subjected to treatment in water and urea for different time (1 5minuites) periods. The area shrinkage percent increases with increasing pretreatment time. It is around $10 \%$ after 5 minutes. 
The effects of microwave pretreatment on the dye ability of wool fabric: Procedure of pretreatment referred to 2.2. The wool fabric to be dyed is padded at about room temperature with the padding liquor which contains Lanasol yellow reactive dye, peregal $\mathrm{O}$, urea, and sodium bi sulfite. The liquor pickup can be $100 \%$ on weight of dry fiber. Thereafter, heating the fabrics in the microwave oven at $700 \mathrm{~W}$ for 6 minuites to fix the dyestuff. Pretreatment process was changed to study the effects of pretreatment on the dye ability of wool fabric. The result was shown in Table 4. The color depth of the soaked in water and urea solution and treated with microwave at various lengths of time are shown in Table 4. It can be seen, from Table 4, that the color depth of wool fabric treated in water and urea by microwave was improved compared to the dyeing color depth of the untreated wool fabric. As expected, water-treatment and urea-treatment causes an obvious rise in wool dye ability. Water and urea are lossy substances, upon exposure to microwave radiation the additional localized energy from oscillation of the water and urea molecules inside the wool fabric may increase the breaking of interaction bonds, causing the internal structure to be opened to greater dye diffusion. It can be seen that pretreatment time has a significant impact on the color depth of the dyed wool fabric. The longer the pretreatment, the greater the color depth of the dyed wool fabric. From the analysis of results obtained with Table 4 it also can be seen that the wool fabric are subjected to the same conditions of pretreatment and dyeing, except pretreatment medium when using urea as a pretreatment media, the dye uptake is much higher than the corresponding ones with an aqueous pretreatment. Urea has a higher dipole moment than water; the increased concentration of urea in pretreatment caused more absorption of microwave radiation. ${ }^{9}$ Thus, there appeared to be a correlation between the concentration of urea and the color depth of dyed wool fabric attained during microwave pretreatment. The higher the concentration of urea, the greater the color depth of the dyed wool fabric.

Table I Influence of microwave heating time on the color depth of dyed wool fabric

\begin{tabular}{|c|c|c|c|c|c|c|c|}
\hline Reactive dyes & $\begin{array}{l}\text { Batching } \\
24 \mathrm{~h}\end{array}$ & $\begin{array}{l}\text { Heating } \\
\text { I min }\end{array}$ & $\begin{array}{l}\text { Heating } \\
2 \mathrm{~min}\end{array}$ & $\begin{array}{l}\text { Heating } \\
4 \mathrm{~min}\end{array}$ & $\begin{array}{l}\text { Heating } \\
6 \mathrm{~min}\end{array}$ & $\begin{array}{l}\text { Heating } \\
8 \mathrm{~min}\end{array}$ & $\begin{array}{l}\text { Heating } \\
\text { I0min }\end{array}$ \\
\hline Lanasol red $(\mathrm{K} / \mathrm{S})$ & 2.0154 & $\mathrm{I} .7047$ & 1.8876 & 1.9029 & 2.0162 & 1.9784 & 1.7123 \\
\hline Lanasol blue $(\mathrm{K} / \mathrm{S})$ & 2.4116 & 2.3639 & 2.3714 & 2.3811 & 2.5686 & 2.5082 & 2.417 \\
\hline Lanasol yellow (K/S) & 1.9933 & 1.8429 & 1.9491 & 1.9532 & 2.0154 & 1.9396 & 1.9114 \\
\hline
\end{tabular}

Table 2 Influence of batching time on the color depth of dyed wool fabric

\begin{tabular}{|c|c|c|c|c|c|c|c|}
\hline Reactive dyes & $\begin{array}{l}\text { Heating } \\
6 \mathrm{~min}\end{array}$ & $\begin{array}{l}\text { Batching } \\
\text { I0min }\end{array}$ & $\begin{array}{l}\text { Batching } \\
20 \mathrm{~min}\end{array}$ & $\begin{array}{l}\text { Batching } \\
\text { 30min }\end{array}$ & $\begin{array}{l}\text { Batching } \\
40 \mathrm{~min}\end{array}$ & $\begin{array}{l}\text { Batching } \\
50 \mathrm{~min}\end{array}$ & $\begin{array}{l}\text { Batching } \\
60 \mathrm{~min}\end{array}$ \\
\hline Lanasol red (K/S) & 2.0162 & 2.0647 & 2.0976 & 2.1229 & 2.1765 & 2.1984 & 2.2223 \\
\hline Lanasol blue (K/S) & 2.5686 & 2.5939 & 2.6314 & 2.6811 & 2.7286 & 2.7682 & 2.787 \\
\hline Lanasol yellow (K/S) & 2.0154 & 2.0429 & 2.0691 & 2.0932 & 2.1354 & 2.1796 & 2.2014 \\
\hline
\end{tabular}

Table 3 Influence of microwave pretreatment on the area shrinkage of wool fabric

\begin{tabular}{llllll}
\hline & $\mathbf{I} \mathbf{m i n}$ & $\mathbf{2} \mathbf{m i n}$ & $\mathbf{3} \mathbf{m i n}$ & $\mathbf{4} \mathbf{m i n}$ & $\mathbf{5} \mathbf{m i n}$ \\
\hline Water & $1.43 \%$ & $2.78 \%$ & $4.17 \%$ & $8.44 \%$ & $10.50 \%$ \\
$50 \mathrm{~g} / \mathrm{L}$ Urea & $1.85 \%$ & $4.02 \%$ & $6.60 \%$ & $8.83 \%$ & $10.70 \%$ \\
I00g/L Urea & $1.95 \%$ & $4.13 \%$ & $7.86 \%$ & $8.89 \%$ & $10.90 \%$ \\
\hline
\end{tabular}

Table 4 Influence of microwave pretreatment on the color depth of dyed wool fabric

\begin{tabular}{lllllll}
\hline & $\mathbf{0 m i n}$ & $\mathbf{I} \mathbf{m i n}$ & $\mathbf{2 m i n}$ & $\mathbf{3} \mathbf{m i n}$ & $\mathbf{4 m i n}$ & $\mathbf{5 m i n}$ \\
\hline Water & 2.0154 & 2.0187 & 2.0197 & 2.0211 & 2.0212 & 2.0217 \\
$50 \mathrm{~g} / \mathrm{L}$ Urea $(\mathrm{K} / \mathrm{S})$ & 2.0154 & 2.0493 & 2.0949 & 2.142 & 2.1602 & 2.1794 \\
$100 \mathrm{~g} / \mathrm{L}$ Urea $(\mathrm{K} / \mathrm{S})$ & 2.0154 & 2.0499 & 2.0876 & 2.1231 & 2.1786 & 2.2317 \\
\hline
\end{tabular}

The effects of microwave pretreatment on the surface morphology of wool fabric: Soaking the wool fabrics in water, $50 \mathrm{~g} / \mathrm{L}$ urea and $100 \mathrm{~g} / \mathrm{L}$ solution at ambient temperature, until the fabrics were completely wetted; heating the fabrics in the microwave oven at $700 \mathrm{~W}$ for 5minutes. Figure 1-4 shows surface structure of the untreated and treated wool samples. It was clear, from Figure 4, that microwave pretreatment in $100 \mathrm{~g} / \mathrm{L}$ urea solution has light damage on surface scale like structure of wool compared to untreated wool fabric. It was considered that the damage of surface scale like improved the absorption of dye molecules by the wool fabric during padding and the diffusion of the dye molecules into the wool fabric. As a result, the probability of the reaction between the reactive dyes and the wool fabric increased, resulting in the improved color depth of the dyed wool fabric. It also can seen, from (Figure 2) (Figure 3), that treatment with water and $50 \mathrm{~g} / \mathrm{L}$ urea did not cause obvious change in surface scale like structure of wool fabric.

The effects of microwave pretreatment on the crystallinity of wool fabric: Procedure of pretreatment referred to 3.3.3. Figure 5-8 showed results of the analysis of the crystallinity of untreated and treated wool samples. It is clear, from Figure 5-8, that the crystallinity of the treated wool fabric were very similar to those of the untreated wool fabric. In other words, the microwave pretreatment did not significantly alter 
the crystallinity of wool fabric. Now as the degree of crystallinity almost unchanged, but in our study the dye uptake increased. This may be due to the following reasons:

a. Breaking down a certain amount of hydrogen bonds and separating molecular chains with thereby increase spacing, size and pores and voids in wool structure, which improves dye ability.

b. The morphological structure of the wool fiber is partially destroyed, which promote the adsorption and permeation of the dye molecules into the wool fibres, again improving the extent of reaction between the reactive dye and the wool fibres.

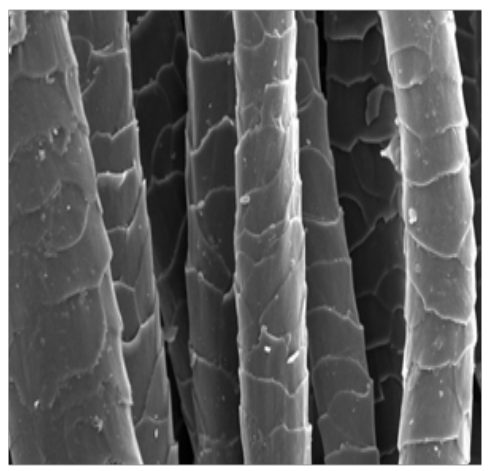

Figure I Untreated wool fabric.

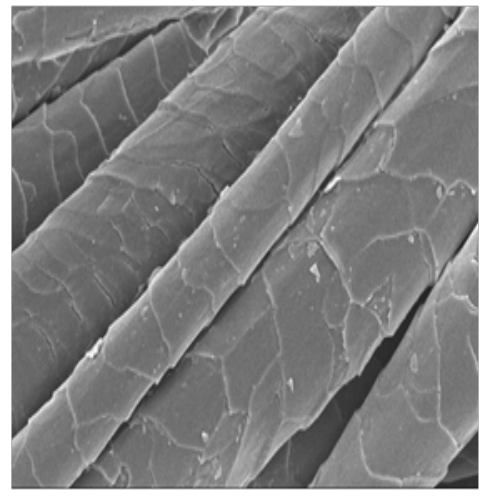

Figure 2 Treatment in water for $5 \mathrm{~min}$.

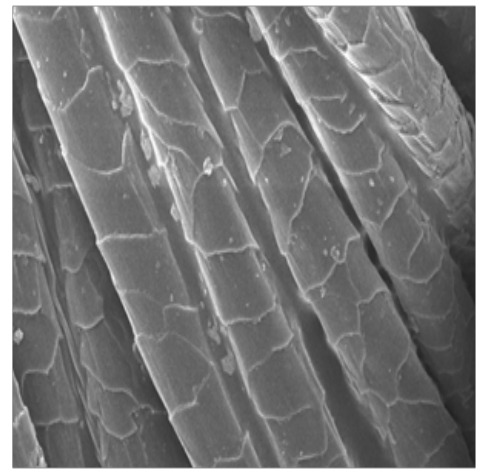

Figure 3 Treatment in 50g/L urea for $5 \mathrm{~min}$.

\section{Dyeing result}

The dyeing procedure of wool fabric involved the following (1) The wool textile to be dyed is padded at about room temperature; the moist cloth is batched at about room temperature for 24 hours, which is customarily effected in the rolled-up state by slowly rotating the beam to fix the dyestuff. (2) The wool textile to be dyed is padded at about room temperature, heating the fabrics in the microwave oven at $700 \mathrm{~W}$ for 6 minuites to fix the dyestuff. (3) Soaking the wool fabrics in water at ambient temperature, until the fabrics were completely wetted; heating the fabrics in the microwave oven at $700 \mathrm{~W}$ for 5 minutes. The wool textile to be dyed is padded at about room temperature, heating the fabrics in the microwave oven at $700 \mathrm{~W}$ for 6 minuites to fix the dyestuff. (4) Soaking the wool fabrics in $50 \mathrm{~g} / \mathrm{L}$ urea at ambient temperature, until the fabrics were completely wetted; heating the fabrics in the microwave oven at $700 \mathrm{~W}$ for 5 minutes. The wool textile to be dyed is padded at about room temperature, heating the fabrics in the microwave oven at $700 \mathrm{~W}$ for 6 minuites to fix the dyestuff. (5) Soaking the wool fabrics in $100 \mathrm{~g} / \mathrm{L}$ urea at ambient temperature, until the fabrics were completely wetted; heating the fabrics in the microwave oven at $700 \mathrm{~W}$ for 5 minutes. The wool textile to be dyed is padded at about room temperature, heating the fabrics in the microwave oven at $700 \mathrm{~W}$ for 6 minuites to fix the dyestuff.

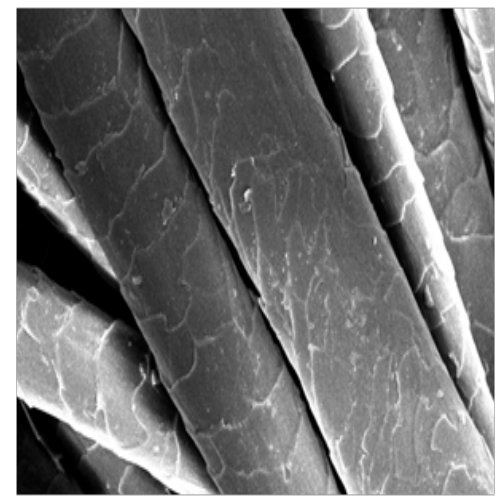

Figure 4 Treatment in 100g/L urea for $5 \mathrm{~min}$.

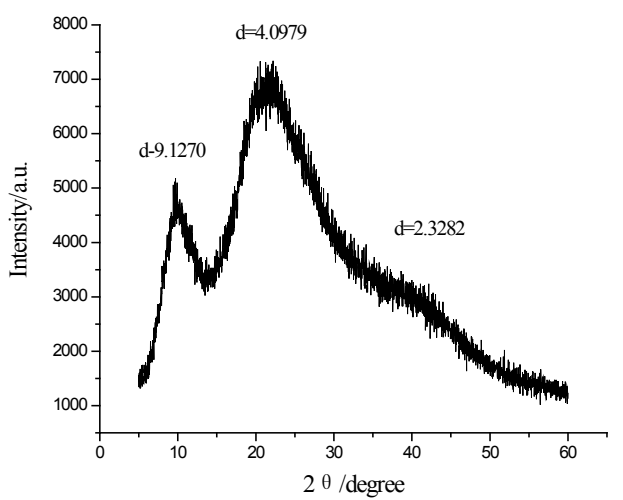

Figure 5 Untreated wool fabric.

Fixation, dyeing uniformity, dye penetration, color fastness, breaking strength and elongation of wool fabrics were compared among cold batching fixation, microwave fixation and microwave fixation after pretreatment.

Fixation and dye uniformity: Some of the results obtained are given in Table 5, in which some cold batching fixation and microwave fixation results are included for comparison. It can be seen that in fixation achieved in the microwave batch after 6 minutes significantly less than that achieved after batching for 24 hours at room temperature. A comparison of the $\mathrm{S}, \mathrm{P}$ and $\Delta \mathrm{E}$ values cold batching fixation and 
microwave fixation showed higher uniformity were achieved than cold batching fixation because the mechanism of microwave heating, in that water vaporization takes place internally. Uneven dyeing was obtained with microwave pretreatment. The factor that may have caused the uneven dyeing could be no uniformity in the application the water and urea when it was soak-applied to the wool fabric and uneven steaming from water bath during microwave exposure.

Color fastness: Table 6 showed that cold batching fixation and microwave fixation all exhibited significantly higher colorfastness to crocking and colorfastness to laundering, which were all above 4 grades.

Table 5 Fixation and dye uniformity of dyed wool fabric
Breaking strength and elongation: Note: Warp breaking strength and elongation of untreated wool fabric were $1169.8 \mathrm{~N}$ and $61.2 \mathrm{~mm}$. Weft breaking strength and elongation of untreated wool fabric were $476.8 \mathrm{~N}$ and $44.3 \mathrm{~mm}$. Changes were studied in breaking strength and elongation of wool fabric subjected to five dyeing procedures is presented in Table 7. It was observed that strength properties and elongation of wool fabric subjected to cold batching fixation, microwave fixation and microwave pretreatment and fixation were slightly changed. Five dyeing procedures had no significant effect on breaking strength and elongation of wool fabric.

\begin{tabular}{|c|c|c|c|c|c|c|c|c|}
\hline \multirow[t]{2}{*}{ Dyeing procedure } & \multirow[t]{2}{*}{ Fixation/\% } & \multicolumn{3}{|l|}{$\mathbf{S}$} & \multicolumn{3}{|l|}{$\mathbf{P}$} & \multirow[t]{2}{*}{$\Delta \mathbf{E}$} \\
\hline & & $\Delta \mathbf{L}$ & $\Delta \mathbf{C}$ & $\Delta \mathbf{H}$ & $\Delta \mathbf{L}$ & $\Delta \mathbf{C}$ & $\Delta \mathbf{H}$ & \\
\hline (1) & 88.9 & 0.28 & 0.35 & 0.07 & 1.04 & 1.03 & 0.12 & 0.59 \\
\hline (2) & 79.2 & 0.3 & 0.43 & 0.09 & 0.84 & 0.6 & 0.2 & 0.47 \\
\hline (3) & 84.6 & 0.59 & 0.43 & 0.28 & 0.69 & 0.76 & 0.39 & 0.85 \\
\hline (4) & 80.2 & 0.51 & 0.5 & 0.17 & 0.65 & 0.97 & 0.26 & 0.78 \\
\hline (5) & 77.8 & 0.4 & 0.52 & 0.14 & 0.73 & 0.83 & 0.25 & 0.69 \\
\hline
\end{tabular}

Table 6 Color fastness of dyed wool fabric

\begin{tabular}{|c|c|c|c|c|c|c|c|c|c|}
\hline \multicolumn{3}{|c|}{ Colorfastness to crocking } & \multicolumn{7}{|c|}{ Colorfastness to laundering } \\
\hline \multirow[t]{2}{*}{ Dyeing procedure } & \multirow[t]{2}{*}{ Dry } & \multirow[t]{2}{*}{ Wet } & \multirow[t]{2}{*}{ Color change } & \multicolumn{6}{|c|}{ Staining on multi fiber } \\
\hline & & & & Wool & Acrylic & Polyester & Nylon & Cotton & Acetate \\
\hline (1) & 5 & 5 & 5 & 5 & 5 & 5 & 5 & 5 & 5 \\
\hline (2) & 5 & 5 & 4 & $4 \sim 5$ & 4 & 5 & 5 & 5 & $4 \sim 5$ \\
\hline (3) & 5 & $4 \sim 5$ & 4 & $4 \sim 5$ & 5 & 5 & $4 \sim 5$ & 5 & $4 \sim 5$ \\
\hline (4) & 5 & $4 \sim 5$ & 4 & $4 \sim 5$ & $4 \sim 5$ & 5 & 5 & 5 & 5 \\
\hline (5) & 5 & $4 \sim 5$ & 5 & 4 & 5 & 5 & $4 \sim 5$ & 5 & 5 \\
\hline
\end{tabular}

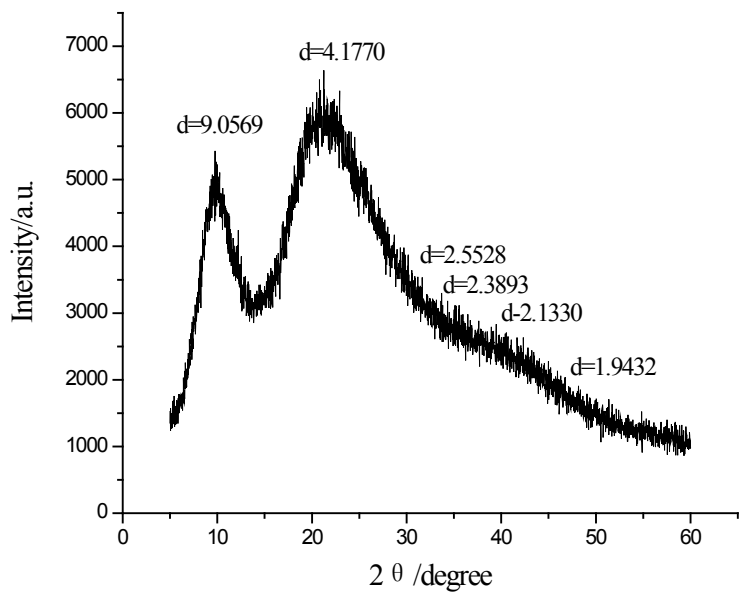

Figure 6 Treatment in water for $5 \mathrm{~min}$.

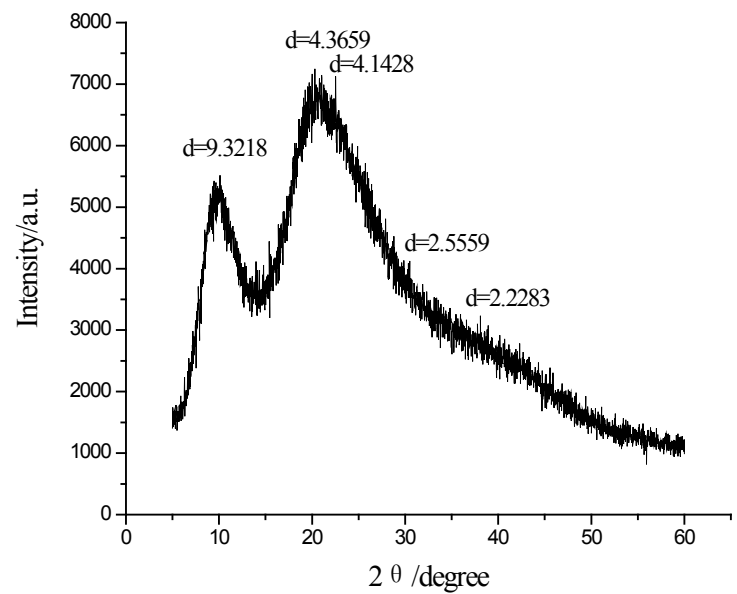

Figure 7 Treatment in 50g/L urea for $5 \mathrm{~min}$. 


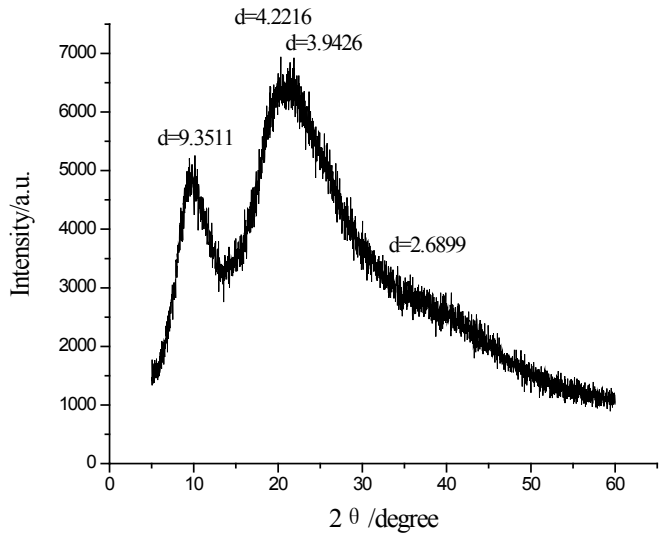

Figure 8 Treatment in 100g/L urea for $5 \mathrm{~min}$.

Table 7 Breaking strength and elongation of dyed wool fabric

\begin{tabular}{lllll}
\hline & \multicolumn{2}{l}{ Breaking strength /N } & \multicolumn{2}{l}{ Breaking elongation $/ \mathbf{m m}$} \\
\cline { 2 - 5 } & Warp & Weft & Warp & Weft \\
\hline (1) & 1023.7 & 453.3 & 60 & 42.1 \\
(2) & 1027.5 & 451.5 & 58.1 & 41.7 \\
(3) & 1021.8 & 452.1 & 60.3 & 42.6 \\
(4) & 1013 & 448.7 & 60.9 & 42.9 \\
(5) & 994.2 & 437.2 & 61.1 & 43.7 \\
\hline
\end{tabular}

\section{Conclusion}

It can be concluded from the investigated report that the rate of dyeing with microwave heating is much faster than cold pad batching, microwave heating for 6 minutes produced better dye fixation results than those obtained after 24 hours batching at room temperature. The color depth of dyed wool fabric was increased when batching for period of time before microwave fixation, and the relative color strength $(\mathrm{K} / \mathrm{S})$ increases by increasing the batching time before microwave fixation.

Microwave pretreatment can improve the dyability of wool fabric. The longer the pretreatment, the greater the color depth of the dyed wool fabric. Wool fabric are subjected to the same conditions of pretreatment and dyeing, except pretreatment medium when using urea as a pretreatment media, the dye uptake is much higher than the corresponding ones with an aqueous pretreatment. The area shrinkage percent increases with increasing pretreatment time. $100 \mathrm{~g} / \mathrm{Lurea} /$ microwave pretreatment also caused the damage of surface scale like structure of wool fiber promoting the adsorption and permeation of dye molecules into the wool fiber, again improving the extent of reaction between the reactive dye and the wool fabric. Microwave pretreatment did not seem to influence the crystallinity of the wool fiber significantly.
Fixation achieved in the microwave batch after 6minutes significantly less than that achieved after batching for 24 hours at room temperature. Microwave fixation showed higher uniformity and dye penetration than cold batching fixation. Uneven dyeing was obtained with microwave pretreatment. Cold batching fixation, microwave fixation and microwave pretreatment and fixation caused only slight reduction in the breaking strength and elongation of wool fabric, which should not have any significant implication on application of the dyed wool fabric. Microwave pretreatment and dyeing technique has significant potential for Industrial application as microwave is a clean, environmentally friendly heating technology.

\section{Disclosure statement}

No potential conflict of interest was reported by the author.

\section{Funding}

This work was supported by Scientific Research Start-up Foundation of Shaoxing University [grant number 20135008].

\section{Acknowledgments}

None.

\section{Conflict of interest}

Author declares there is no conflict of interest in publishing the article.

\section{References}

1. Oishanskii AI, Oishanskiik VI, Zhernosek SV. Moisture exchange in drying natural fabrics in a microwave electromagnetic field. $J$ Engineering Physics \& Thermo physics. 2013;86(5):1108-1116.

2. Haghi AK. A Study on Microwave Heating. Computational mechanics. 2004;(9):5-10.

3. Yat CS, James KH, Jukka PM. A New Approach to Cure and Reinforce Cold-Cured Acrylics. Silicon. 2012;4(3):209-220.

4. Zhao Xue, He Jinxin, Min Jie. Dyeing behaviors of wool fabric with microwave/hydrogen peroxide treatment. Dyeing and Finshing. 2010;(3):7-10.

5. Delan MJ. Pad-Batch-Microwave Dyeing of Wool. Pigment \& Resin Technology. 1972;4(5):29-32.

6. Zhao Xue, Wang Hongli, He Jinxin. The effect of chlorination pretreatment on the dyeing behavior of wool. Melliand China. 2010;(4):31-32.

7. Zhao Xue. Study of dyeing properties of wool fabrics treated with microwave. The J Textile Institute. 2016;107(2):258-263.

8. Yoshimura Y, Ohe T, Ueda M, et al. Effect of microwave heating on dyeing. Seni Gakkaishi. 2007;63(6):146-151.

9. NSE Ahmed, RM El-Shishtawy. The use of new technologies in coloration of textile fibers. J materials science. 2010;45(5):1143-1153. 